\title{
NO SOMOS EFICIENTES CUANDO SE TRATA DE NEGOCIAR. APROXIMANDO BREVEMENTE A LOS ABOGADOS A UN PROCESO EFECTIVO DE NEGOCIACIÓN
}

\author{
Ricardo Padilla Parot \\ Universidad Diego Portales
}

\section{INTRODUCCIÓN}

No es extraño escuchar en la práctica legal proverbios que aducen que un "mal acuerdo es mejor que un buen juicio", o declaraciones políticas como aquellas realizadas por la exprimer ministra británica Theresa May, señalando -en un acalorado contexto de Brexit- que "no llegar a acuerdo es mejor que un mal acuerdo". ¿Pero resulta esto cierto? Desde el punto de vista de la litigación, es posible argumentar que el mencionado proverbio no constituye la regla general. En efecto, cuando las posibilidades resultan ciertas para una de las partes disputantes o las acciones deducidas son notoriamente frívolas, llegar a un arreglo no parece una solución razonable. Lo mismo es posible decir respecto de acuerdos que contienen propuestas que no coinciden con los intereses que las partes tienen en vista para alcanzar un arreglo. Luego, pero ahora asumiendo que existen buenas razones para negociar antes de entrar al campo de batalla ${ }^{1}$, o golpear y abandonar la mesa sin una solución

\footnotetext{
${ }^{1}$ ¿Por qué preferir un acuerdo en lugar de un juicio? La disputa entre Buchwald y Bernheim con Paramount Pictures relativa a la película Coming to America, ha sido entregada como un clásico ejemplo en los textos sobre negociación. En el caso, luego de tres años de litigación, ambas partes estimaron salir victoriosas. Los demandantes señalaron haber ganado cerca de un millón de dólares y haber hecho incurrir a Paramount en casi tres millones de dólares en su defensa. Por su lado, la demandada señaló que la condena representaba solo una fracción de los USD 6200000 que los demandantes reclamaron originalmente. Sin embargo, y a pesar de que Art Buchwald obtuvo una sentencia gananciosa por USD 150000 para su parte, este había incurrido en gastos procesales por USD 200 000. Tiempo después, Art Buchwald tras obtener este resultado que ni siquiera logró cubrir el costo del juicio, declaró que: "esperaba estar envuelto en un proceso que se resolviera tempranamente por una suma mínima de dinero y, ojalá, una disculpa (...). Uno de los descubrimientos de una demanda como esta es que te lastima profundamente, y uno no olvida con facilidad (...). No cuentes con recibir dinero en una demanda (...) esto resulta cierto ya sea ganes o pierdas". MnOokin \& Ross (1995), pp. 4-5. No obstante, existen diversos casos en que negociar y llegar a un acuerdo resultan difíciles. A veces existe el interés de una parte en la creación de un precedente jurisprudencial, el cuestionar una doctrina legal existente o lograr una reivindicación de connotación pública. En otras ocasiones, existe un fin corporativo en el hecho de deducir una demanda, por ejemplo, con el objetivo de demostrar la validez de derechos de propiedad intelectual que constituyen el centro del negocio o establecer la reputación de que no se cederá con facilidad y así desincentivar la litigación. MNOOKIn et al. (2000), pp. 107 y 225.
} 
negociada, ¿ ¿por qué el resultado de una negociación tiene que ser necesariamente un acuerdo inadecuado para las partes?

La negociación es un proceso utilizado para obtener lo que uno quiere de otros $^{2}$. El problema es que normalmente los negociadores no saben de qué manera aproximarse a dicho proceso, ni cómo definir de manera correcta sus intereses ante un posible acuerdo. De hecho, a pesar de que las partes logren acordar una solución que podría estimarse como un intercambio equitativo, dicha solución habitualmente no será eficiente, porque no tomará en consideración todas las ventajas que traen los intereses contrapuestos existentes entre ellas ${ }^{3}$.

En efecto, existe evidencia científica que prueba que solo el 3\% de los negociadores logran llegar a un acuerdo en que todos ganan (win-win deals) ${ }^{4}$. A la inversa, el 20\% de las partes negociantes -aún con un importante grado de experiencia en estos procesos- optan, paradójicamente, por un arreglo en que todos saldrán perdiendo (lose-lose outcomes), es decir, un quinto de los negociadores llegará al peor de los resultados posibles ${ }^{5}$. En suma, se ha concluido que más de un $90 \%$ de los negociadores habrá desperdiciado la oportunidad de obtener un mejor acuerdo que el adoptado en los hechos ${ }^{6}$. De esta manera, se encuentra comprobado que el normal desempeño de las partes en un proceso de negociación será contraproducente. El resultado de la negociación no solo se traducirá en soluciones subóptimas, sino que, 120 también, muchas veces causarán un mayor daño a la relación existente entre los disputantes y dejará sin resolver conflictos donde existían soluciones que satisfacían de mejor manera a los intereses de las partes, en contraste con una lucha prolongada. Realmente alarmante.

El presente trabajo es un modesto aporte al estado actual que en el ámbito comparado dispone la ciencia y estudio de la negociación y de las implicancias que tienen sobre los negociadores diversos procesos cognitivos. Pero la escasez de este tipo de textos en nuestro país demuestra una falta de interés por el estudio profundo y práctico de una de las disciplinas a la que se ven enfrentados a diario los abogados - pero también toda otra clase de personas, pues negociar es una cuestión realmente rutinaria- y en la cual nos encontramos hoy fallando dramáticamente.

Este artículo, entonces, se limita a explicitar y dar conciencia de algunas de las razones por las cuales los abogados negociamos de manera ineficiente. Y para ello, la tarea que sigue es doble: primero, se desarrollarán las razones por las cuales deberíamos escoger negociar y determinar, dentro de ese pro-

${ }^{2}$ Fisher \& Ury (2012), p. xxv.

${ }^{3}$ MnOokin \& Ross (1995), p. 9. Los citados autores agregan que en muchos de los acuerdos que las partes logran cerrar -ya sea por negociación o imposición- estas no logran satisfacer los criterios de eficiencia económica, op. cit., p. 3.

${ }^{4}$ NAdLer et al. (2003), p. 535.

${ }^{5}$ Thompson \& Hrebec (1996), p. 403.

${ }^{6}$ Op. cit., p. 400. 
ceso, la gama de posibles soluciones a ser obtenidas. Segundo, se analizarán las causas por las que a pesar de que somos conscientes de nuestros intereses y objetivos en conflicto, aun así, fallamos en acordar en una solución que pueda satisfacernos de manera adecuada. En consecuencia, en esta segunda parte serán abordados algunos de los problemas relacionados a la errónea percepción que surge sobre la persona y actos de la contraparte, y que afectan a los procesos de creación y distribución de ganancias a ser realizados una vez sentados en la mesa negociadora. Finalmente, se sugerirán brevemente tres posibles herramientas o técnicas para manejar aquellos problemas, las que tienen por objetivo construir cooperación y confianza entre las partes.

\section{El objetivo de LA NEGOCiACIÓN:}

LA BÚSQUEDA DE UN ACUERDO QUE DEJE A LAS PARTES MEJOR POSICIONADAS

La negociación ha sido definida como un proceso de comunicación diseñado para llegar a un acuerdo entre dos o más partes, las que mantienen algunos intereses que son compartidos y otros que se encuentran en conflicto o, simplemente, difieren ${ }^{7}$. La razón por la cual negociamos se basa en la idea de producir un resultado mejor que el posible de obtener sin el acto de negociar. En consecuencia, el objetivo de la negociación no es necesariamente llegar a un acuerdo, porque un acuerdo solo tendrá sentido si deja a las partes mejor posicionadas ${ }^{8}$.

\section{A. Un acuerdo en el que todos ganan: la eficiencia en el proceso de negociación}

¿Pero cómo se determina aquella mejor posición? Lo primero a señalar es que normalmente las personas no se enfocan en lo que quieren. Al contrario, las personas se centran más en la manera de obtener las cosas y olvidan sus propias motivaciones o intereses. En este sentido, es de suma importancia evitar lo que ha sido denominado como errores del tercer tipo, los que se traducen en resolver el problema equivocado. En efecto, la primera tarea a realizar por alguien que debe tomar una decisión, es desafiar su entendimiento sobre las opciones a las que se está enfrentando. Debe ser examinado, entonces, la razón o causa por la cual una decisión debe ser tomada. Esta causa, o la definición del problema, dependerá de los objetivos, intereses y deseos que se quieren satisfacer ${ }^{9}$.

Determinadas las motivaciones tras el conflicto, un contraste de ellas debe realizarse con lo que Roger Fisher y William Ury han definido bajo el acrónimo anglosajón BATNA: la mejor alternativa a un acuerdo negociado. El BATNA

\footnotetext{
${ }^{7}$ Patton (2005), p. 279.

${ }^{8}$ Op. cit., p. 285.

${ }^{9}$ RAIFFA et al. (2007), pp. 16-17.
} 
constituye aquella alternativa ${ }^{10}$-ya sea que se traduzca en inactividad, retardo, entorpecimiento, demandar, cerrar con un tercero, entre tantas otras estrategias que se ven en la práctica legal- que satisface mejor (dentro del espectro de posibles soluciones existentes) los intereses de una parte negociadora, y que, en efecto, tomará de no ser posible llegar a un acuerdo con la contraparte ${ }^{11}$. El BATNA, de esta forma, constituye aquel estándar con el que debe contrastarse cualquier propuesta de acuerdo, pues será, en definitiva, lo único que protegerá a un negociador de aceptar términos que son desfavorables o de rechazar arreglos que pueden estar en el interés de aquel aceptar ${ }^{12}$.

Negociar, entonces, no implica renunciar a todo lo que es importante para uno. Solo se requiere encontrar la voluntad de sentarse con el adversario y determinar si es posible llegar a un acuerdo que sirva al interés de las partes de mejor forma de lo que sus BATNA podría hacerlo ${ }^{13}$. Si un acuerdo así es posible, las partes, luego del proceso de negociación, obtendrán un buen resultado, dado que aquel acuerdo será considerado eficiente y, probablemente, mejorará -o al menos no dañará- la relación entre ellas ${ }^{14}$. Sin embargo, debe enfatizarse que un acuerdo será posible y sostenible en el tiempo (no será repudiado con posterioridad) solo si también cumple con los intereses de la otra parte, al menos, tan bien como su propio BATNA ${ }^{15}$.

Sin embargo, conocer el BATNA no es el paso final. Aquel debe ser traducido por los negociadores en un RV, es decir, en aquella cantidad que 122 a cada parte le es indiferente alcanzar mediante un acuerdo o retirarse de las negociaciones con sus respectivos BATNA. Cuando ambas partes han determinado sus RV, es luego posible establecer una ZOPA, la que consiste en la extensión del superávit formado entre el RV de los negociadores, que puede ser luego dividido entre ellos. En suma, cualquier acuerdo alcanzado dentro de la ZOPA dejará a las partes mejor posicionadas que sin cerrar un acuerdo ${ }^{16}$. De allí que es posible decir que el objetivo de la negociación es precisamente generar valor o ganancias para las partes. Si aquello no es posible, simplemente no vale la pena negociar.

${ }^{10}$ Las alternativas pueden ser traducidas en aquel rango de cosas que es posible hacer fuera de la mesa de negociación y sin contar con el acuerdo de la otra parte. MNOOKIN et al. (2000), p. 19.

${ }^{11}$ Mnookin et al. (2000), p. 19.

${ }^{12}$ Fisher \& URY (2012), p. 102.

${ }^{13}$ Mnookin (2011), p. 369.

${ }^{14}$ Fisher \&URY (2012), p. 4.

${ }^{15}$ Siguiendo a Bruce Patton, un buen resultado consistirá en una solución creativa, elegante y sin desperdicios; que logre capturar la mayor cantidad de ganancias posibles. Dicha solución debe ser a su vez legítima, con la cual nadie sienta que han tomado provecho de ella. Un buen resultado debe traducirse en un compromiso firme, implementable y sostenible; alcanzado tras un proceso lo más eficiente posible mediante una buena comunicación; un proceso que, en efecto, ayude a construir precisamente el tipo de relación que queremos con la contraparte. Patton (2005), pp. 285-286.

${ }^{16}$ Mnookin et al. (2000), pp. 19-20. 


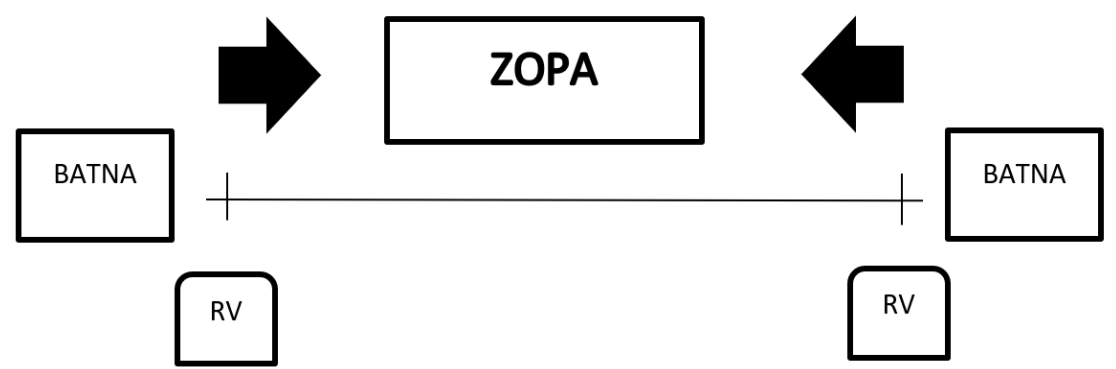

De este modo, un buen acuerdo se tornará en el resultado de una negociación que beneficiará a las partes, cuando todas las alternativas han sido evaluadas y no se ha dejado ningún recurso sobre la mesa ${ }^{17}$. Siguiendo a Leigh Thompson, existen tres niveles de acuerdos que resultarán beneficiosos para las partes (win-win agreements). El primero se logra cuando los negociadores obtienen un acuerdo que comprende un mejor resultado que sus RV. En el segundo nivel es posible encontrar aquellos arreglos que son mejores (o más deseables) para ambas partes que otros posibles acuerdos disponibles dentro de la ZOPA. Finalmente, en el tercer nivel, se ubican aquellos acuerdos que se encuentran dentro de la frontera de un resultado óptimo de Pareto. Es decir, en este último nivel, no existe ninguna alternativa que pueda mejorar la posición de una de las partes sin dañar el resultado de la otra ${ }^{18}$.

\section{B. Todos salen perdiendo: cerrar acuerdos que resultan indeseados}

A la inversa, comportamientos inefectivos en los procesos de negociación pueden conducir a resultados indeseados. Una manifestación de este fenómeno tiene lugar cuando existe una solución que dejará a las partes en una mejor posición que la que en los hechos se ha adoptado. En la literatura este fenómeno ha sido conceptualizado como "soluciones subóptimas" ${ }^{19}$. Dentro de estas, se ha definido a los acuerdos en que todas las partes terminan perdiendo (lose-lose agreements) como aquellos arreglos que fallan en optimizar intereses que son compatibles. En otras palabras, cuando enfrentados a la tarea conjunta de asignar recursos, los negociadores acuerdan en un resultado que es claramente peor para ambos ${ }^{20}$.

Desde luego, los lose-lose agreements solo tendrán lugar cuando las partes tienen más de una alternativa de acuerdo a elegir. Precisamente, la ineficacia en las negociaciones ocurre cuando los negociadores realizan los procesos

\footnotetext{
${ }^{17}$ Thompson (2012), p. 90.

${ }^{18}$ Op. cit., pp. 92-93.

${ }^{19}$ Thompson \& Hastie (1990), p. 99.

${ }^{20}$ Thompson \& Hrebec (1996), p. 407.
} 
cognitivos de "juzgar" y "escoger". En este contexto, el término juzgar se refiere a los aspectos cognitivos del proceso de toma de decisión. En efecto, si no se tienen alternativas a elegir, no se tiene una decisión que tomar ${ }^{21}$. Así, la tarea de juzgar implica la evaluación realizada por el negociador respecto del contenido de las soluciones que se encuentran a su alcance ${ }^{22}$. Por otro lado, la tarea de escoger implica que el negociador debe determinar-luego de juzgar- cuál de las opciones de arreglo prefiere ${ }^{23}$.

Científicos han demostrado que los lose-lose agreements constituyen una realidad que resulta bastante habitual, estableciendo que, dentro de los procesos cognitivos de juzgar y escoger, los negociadores tienden a no reconocer cuando entre estos existen intereses en juego que son compatibles, acordando en arreglos que las partes encuentran indeseables en contraste con otros disponibles sobre la mesa ${ }^{24}$.

\section{Problemas de INEFICIENCIA EN EL PROCESO DE NEGOCIACIÓN}

Leight Thomson y Dennis Hrebec lo han señalado claramente: la sola existencia de acuerdos en que todas las partes pierden, implica, necesariamente, la existencia de acuerdos en que ambas partes pueden llegar a obtener resultados en que todas queden mejor posicionadas ${ }^{25}$. Pero a pesar de ello, 124 como fuera dicho, los negociantes tienden a llegar a resultados indeseados. Existen, en efecto, diversas barreras que inhiben a los negociadores lograr el objetivo del proceso de negociación, causando el denominado "problema de rentabilidad eficiente en la resolución de disputas" ${ }^{26}$. En la literatura pueden ser encontrados varios de estos obstáculos ${ }^{27}$, sin embargo, no es posible aquí explorar todos aquellos. En su lugar, serán desarrollados algunos de los problemas que surgen en las etapas de creación y distribución de ganancias de un proceso de negociación.

${ }^{21}$ Bazerman \& MoOre (2013), pp. 1-2.

${ }^{22}$ Korobkin \& Guthrie (2006), p. 328.

${ }^{23}$ Ibid.

${ }^{24}$ Thompson \& Hrebec (1996), pp. 403,404. Siguiendo a Leigh Thompson, la razón de la existencia de los acuerdos en que todas las partes pierden no se debe a falta de motivación o inteligencia. El problema se origina en cuatro sesgos psicológicos: egocentrismo, procesamiento de información confirmatoria, satisfacción e incompetencia autorreforzante. THOMPSON (2012), pp. 26-27.

${ }^{25}$ Thompson \& Hrebec (1996), p. 407.

${ }^{26}$ MnOokin \& Ross (1995), p. 7.

${ }^{27}$ El libro Barreras a la Resolución de Conflictos (Barriers to conflict resolution) del Centro de Conflicto y Negociación de la Universidad de Stanford, entrega tres categorías de barreras u obstáculos al proceso de negociación: (1) Barreras estratégicas y tácticas, que surgen de los esfuerzos de los negociadores por maximizar sus propios resultados; (2) Barreras psicológicas, las que reflejan los procesos cognitivos o sesgos humanos en la interpretación de la información y (3) Barreras organizaciones e institucionales, las que constituyen diversos factores desde estructuras burocráticas hasta consideraciones políticas. MNOOKIN \& Ross (1995), pp. 3-24. 


\section{A. El dilema del negociador: la tensión entre crear y distribuir ganancias}

Diversos investigadores han presentado al proceso de negociación bajo la analogía de hacer un pastel (o, como se es referido en Chile, una "torta") y luego dividirla ${ }^{28}$. En razón de ello, ha sido explicado que el proceso de negociación involucra el manejo de problemas tanto al dar tamaño a la torta como al determinar quién se quedará con la porción más grande ${ }^{29}$. La encrucijada entre el objetivo de hacer una torta más grande (maximizando el valor conjunto que entrega un acuerdo) y reclamar la porción más grande (maximizando la cuota de cada parte) ha sido denominado el "dilema del negociador" (negotiator's dilemma) ${ }^{30}$. El dilema radica en el hecho de que estrategias diseñadas para agrandar la torta (como lo es el intercambio de in formación entre las partes), pueden entrar en conflicto con estrategias empleadas para reclamar una porción más grande de la misma (actuando a través de engaño o de manera oportunistas ${ }^{31}$. En pocas palabras, un negociador siempre querrá crear de manera conjunta la mayor cantidad de ganancias posibles, reclamar la mayor parte de ellas y evitar, al mismo tiempo, que el otro lado se aproveche y obtenga una mejor porción ${ }^{32}$.

Ha sido argumentado que negociadores mezquinos, preocupados solo de maximizar su porción de la torta, pueden llevar a las partes a resultados ineficientes ${ }^{33}$. Esta conclusión surge del conocido "dilema del prisionero" (o el "dilema de dos personas" como fue originalmente llamado). Se recordará que en el escenario de los dos criminales capturados y encerrados de manera separada sin comunicación, traicionar al compañero rufián ofrece un mejor acuerdo con los fiscales que en la alternativa de mantener silencio y actuar de manera coordinada ${ }^{34}$. Los prisioneros egoístas, preocupados de sus propios intereses, se traicionarán mutuamente. Lo interesante de dicho comportamiento, es que la búsqueda de una recompensa individual lleva a ambos prisioneros a delatar al otro, cuando en la práctica ambos obtendrían una mejor recompensa individual si mantuvieran silencio. El dilema del prisionero, entonces, ha dejado una conclusión científicamente clara: comportamientos racionalmente egoístas y descoordinados, pueden traer consigo pésimos resultados a la mesa de negociación ${ }^{35}$.

${ }^{28}$ Véase, entre otros, por ejemplo, Fisher \& URY (2012); THOMPSON (2012); PATTON (2005); MNOOKIN et al. (2000).

${ }^{29}$ MnOokin (1993), p. 239.

${ }^{30}$ Véase Lax \& Sebenius (1986).

${ }^{31}$ RaIfFA et al. (2007), p. 85.

${ }^{32}$ Lax \& Sebenius (2006), p. 205

${ }^{33}$ Mnookin (1993), p. 241.

${ }^{34} \mathrm{Si}$ ambos prisioneros mantienen silencio, serán condenados a un año de cárcel. Si uno traiciona, y el otro mantiene silencio, el primero quedará en libertad y el segundo será condenado a tres años. Si ambos se traicionan, serán condenados a dos años.

${ }^{35}$ RAIFFA et al. (2007), p. 67. 
De esta manera, para agrandar la torta y generar ganancias o, si se quiere, lograr un acuerdo en que todas las partes puedan ganar, es necesario un esfuerzo conjunto a través del intercambio de información. Sin la revelación de preferencias, recursos y alternativas de cada uno de los negociantes, será difícil encontrar acuerdos que consideren sus intereses para dejarlos mejor posicionados ${ }^{36}$. Sin embargo, el intercambio de información en las negociaciones es una espada de dos filos: esencial para resolver conflictos conjuntamente y generar ganancias, es también una fuente de vulnerabilidad ante una parte que solo busca obtener para sí la mejor porción del acuerdo ${ }^{37}$. Desde este punto de vista, la eficiencia en la negociación puede ser entendida como la relación entre el intercambio de información realizada por las partes y la deseabilidad del resultado obtenido en función de la información revelada ${ }^{38}$.

Finalmente, es importante tener presente que el dilema de los negociadores no puede ser resuelto. Ya sea que las negociaciones sean instantáneas, de largo término o de carácter repetitivas ${ }^{39}$, la tensión entre crear y distribuir ganancias estará siempre presente. La tarea, por tanto, es manejar dicha tensión con el objetivo de crear el máximo de ganancias conjuntas y reducir los riesgos de aprovechamiento por una de las partes al momento de ser distribuidas $^{40}$.

\section{B. Problemas en la creación de ganancias}

Fue establecido que crear ganancias a través del proceso de resolución de controversias y llegar a acuerdos en que todas las partes ganen, es el objetivo principal de la negociación. Sin embargo, existen dos -falsas- suposiciones interrelacionadas que evitan que la torta pueda ser agrandada por negociadores.

\section{El conflicto ilusorio}

Es aquel que no es realmente conflicto, pero los negociadores lo consideran como tal ${ }^{41}$. En otras palabras, este tipo de conflicto ocurre cuando las partes creen que sus intereses son incompatibles, cuando en los hechos, no lo son ${ }^{42}$.

${ }^{36}$ Mnookin et al. (2000), p. 9.

${ }^{37}$ LAX \& Sebenius (2006), p. 206. Se ha agregado que el núcleo de esta tensión se encuentra en el hecho de que, sin el intercambio de información, es difícil crear valor o ganancias. Sin embargo, cuando se realiza por un solo lado de la mesa, aquel negociante arriesga que la contraparte tome ventaja. MNOOKIN et al. (2000), p. 17.

${ }^{38}$ SchWARZ et al. (2010), p. 5.

${ }^{39}$ MnOokin et al. (2000), p. 43; Thompson (2012), p. 28.

${ }^{40}$ MnOokin et al. (2000), p. 27.

${ }^{41}$ Thompson \& Hrebec (1996), p. 407.

${ }^{42}$ Thompson (2012), p. 73. Por ejemplo, en el contexto de las negociaciones de Brexit, un conflicto ilusorio surgió luego de una carta enviada en 2017 por Theresa May al presidente del Consejo Europeo Donald Tusk. Conforme al texto de la carta, en términos de seguridad, el fracaso en llegar a un acuerdo significaría que la cooperación entre el Reino Unido y la Unión Europea en la lucha contra el crimen y el terrorismo se vería debilitada. REUTERs (2017). 
Esta suposición de intereses contrapuestos obstaculiza al proceso de creación de ganancias, debido a que hace que los negociadores confundan intereses opuestos con aquellos que son diferentes; siendo esta última clase los que en efecto generan el espacio en que las partes pueden realizar intercambios que les permitirán llegar a acuerdos que las dejaran mejor posicionadas ${ }^{43}$.

Baste recordar que la gran mayoría de los contratos son cerrados precisamente porque las personas no están de acuerdo en el valor o la importancia del objeto del contrato. Nuestro decimonónico Código Civil ya lo entendía así, y de allí que su art. 1441 clasifique en conmutativos a aquellos contratos en que las partes intercambian algo que se "mira como equivalente", pero que subjetiva u objetivamente no lo es. Aunque el proceso de concluir un contrato es disímil a resolver un conflicto, la aproximación a tomar no lo es tanto. En efecto, difícilmente las partes tendrán las mismas preferencias en materias que concurren en una misma negociación, tales como la valuación del objeto en disputa; las expectativas o proyecciones sobre las posibles ganancias a obtener; los tiempos necesarios para resolver el conflicto; las capacidades financieras o la resistencia a los posibles riesgos que involucra el conflicto. Todas aquellas diferencias constituyen verdaderos espacios de intercambios, en que una u otra parte puede entregar, dejar, pedir, transar o converger en favor de la otra.

2. Situaciones de suma cero y la percepción de la existencia de una torta de tamaño invariable

Los negociadores entran al juego con la idea de que las ganancias para una parte constituyen, necesariamente, pérdidas para la otra ${ }^{44}$. En otras palabras, las personas piensan que entre las partes todos los intereses y motivaciones se encuentran en conflicto o son incompatibles: +10 para mi es -10 para ti. Esta tendencia cognitiva ha sido explicada bajo el concepto de situaciones de suma cero o la percepción de la existencia de una torta invariable (fixedpie perception $)^{45}$.

No obstante, la realidad no sigue aquella regla tan estricta. Pocos conflictos son ganar o perder, pero los negociadores no dan cuenta de aquello debido a que

Pero aquella posición de la ex Primera Ministra es errada. Con o sin acuerdo, el interés del Reino Unido y la Unión Europea en la protección de sus ciudadanos jamás permitiría que la cooperación mutua en contra de actos de terroristas disminuya. Políticamente sería inaceptable.

${ }^{43}$ Thompson \& Hastie (1990), p. 101.

${ }^{44}$ Thompson \& Hrebec (1996), p. 407, han explicado que los sistemas cognitivos humanos se basan, ante todo, en una arquitectura de ganar o perder: mis ganancias vienen a costas de la contraparte y viceversa.

${ }^{45}$ Siguiendo a BAZERMAN \& MoOre (2013), pp. 193-194, cuando los individuos se acercan a las negociaciones con una mentalidad de suma cero, suponen que los intereses de uno entran en conflicto directamente con los intereses del otro. La suposición de la existencia de una situación de suma cero lleva a interpretar las cosas de manera más competitiva, como tan solo ganar o perder. 
están concentrados en los aspectos competitivos de la disputa, en lugar de centrar sus esfuerzos en los elementos colaborativos de la misma ${ }^{46}$. Normalmente en un proceso de negociación, las motivaciones de las partes están correlacionadas de manera imperfecta, lo que significa que las ganancias de una parte no representan necesariamente perdidas para la otra ${ }^{47}$. En este sentido, los negociadores realizan un análisis defectuoso sobre las motivaciones del otro lado, el que tiende a exagerar la percepción sobre la existencia de conflictos e incrementar la tendencia a percibir de forma negativa tanto a la persona como las ofertas de la otra parte ${ }^{48}$. Sin embargo, con un análisis ponderativo sobre el objeto de la negociación, los negociadores podrán encontrar una solución adecuada, porque normalmente su evaluación sobre las cosas que están en juego difiere: la misma cosa será menos o más valiosa para la otra parte, permitiéndoles, de esta manera, intercambiarla por otra ${ }^{49}$.

Así, científicos han concluido que los negociadores que han logrado obtener una percepción precisa de los intereses de la otra parte, conducen a negociaciones más beneficiosas ${ }^{50}$. Esto resulta importante, porque al final del día, pensar que resolver el problema de la otra parte es solo "su problema", es lejos de ser el enfoque correcto. Al contrario, para llegar a un acuerdo, es necesario desarrollar una solución que también cumpla con los intereses de la contraparte ${ }^{51}$.

\section{Problemas de distribución de ganancias}

Dará igual que tan grande puedan las partes hacer la torta, si no son capaces de dividir las ganancias creadas en el proceso de negociación. En efecto, debido al deseo de obtener la mayor parte del valor creado, los negociantes puede que, en definitiva, no lleguen a ningún acuerdo a pesar de que aquel pueda beneficiarles. Incluso, si se lograra alcanzar un acuerdo, puede que las partes innecesariamente hayan desperdiciado tiempo y recursos ${ }^{52}$.

Fue señalado que cualquier arreglo adoptado dentro de la ZOPA constituirá un buen acuerdo para las partes. Si eso es cierto, se preguntarán entonces,

${ }^{46}$ RAIFFA et al. (2007), p. 279.

${ }^{47}$ Thompson (2012), p. 94.

${ }^{48}$ RaIFFA et al. (2007), p. 282.

${ }^{49}$ Thompson \& Hastie (1990), p. 100. Se ha señalado que la noción de que las diferencias pueden crear valor o ganancias resulta contraintuitiva. La verdad es que las diferencia son más útiles que las similitudes en ayudar a las partes a llegar a un acuerdo. Las diferencias son el escenario de posibles ganancias a ser intercambiadas, y es a través de ese intercambio que un mayor valor para las partes es creado. Cinco tipos de diferencias -en recursos, valoración, expectativas y preferencias en tiempo y riesgo- son todas potencialmente fuentes de creación de ganancias. MNOOKIn et al. (2000), pp. 14-15.

${ }^{50}$ Thompson \& Hastie (1990), p. 117.

${ }^{51}$ Fisher \&URY (2012), p. 61. Por eso es que se ha enfatizado categóricamente que entre más atención se pone a la posición de una de las partes, menos atención se dedica a satisfacer las preocupaciones subyacentes de los negociadores. FishER \& URY (2012), p. 6.

${ }^{52}$ MNOOKIn et al. (2000), p. 22. 
¿por qué los negociadores fallan en alcanzar un escenario que es relativamente sencillo? Tómese por ejemplo la situación que surge en cualquier compraventa, en que la discusión sobre el precio constituye normalmente el objeto principal de la negociación. El vendedor pensará: Aceptaré al menos nueve millones (RV-BATNA). ¿Cómo obtengo lo más que pueda? ¿Cuánto será lo máximo que el comprador aceptará pagar? ¿Cuál será el menor precio que el comprador pensará estaré dispuesto a aceptar? Por su lado, el comprador pensará: No pagaré más de once millones (VR-BATNA). ¿Qué puedo hacer para pagar lo menos posible? ¿Cuál será el menor precio que el vendedor estará dispuesto a aceptar? ¿Cuál será el máximo precio que el vendedor cree que pagaré? Dentro de la ZOPA del vendedor y comprador, existen dos millones de ganancias a ser distribuidos, sin embargo, es probable que las partes no sepan de su existencia o no logren distribuirla de forma eficiente.

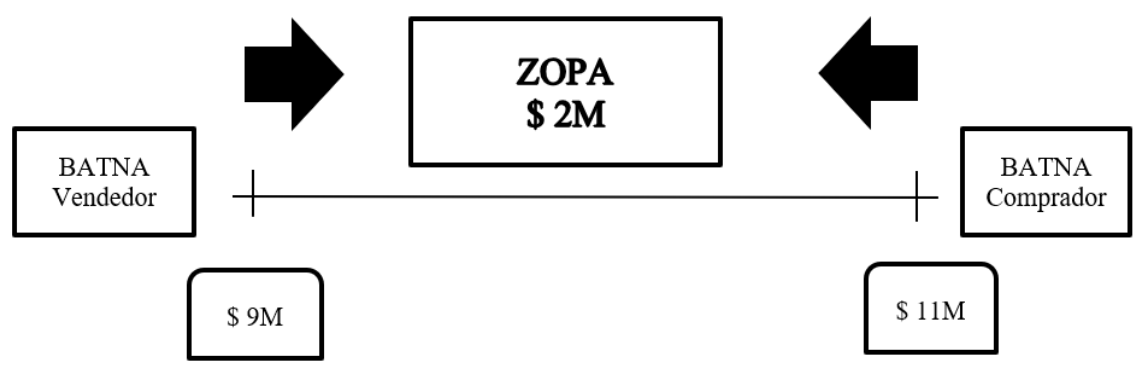

Dos factores han sido indicados como responsables de este fenómeno:

\section{Asimetrías de información}

En casi todos los procesos de negociación, las partes tienen un diferente nivel de información en relación con el objeto transaccional o en disputa. Asimismo, los negociadores son reacios a revelar sus RV y, aunque estuvieran dispuestos a hacerlo, es sumamente difícil para las partes verificar la veracidad de la información proporcionada. Los negociantes, entonces, tendrán un déficit de información, debido a que no estarán en las condiciones de evaluar si aquella es válida ${ }^{53}$.

La falta de completitud en la información genera desconfianza en las ofertas realizadas por la contraparte y aumenta uno de las barreras psicológicas a la resolución de controversias denominada "sesgo de desvalorización reactiva" (reactive devaluation bias). Este sesgo implica que las ofertas realizadas por el otro lado de la mesa serán calificadas negativamente tan solo por aquel hecho. En efecto, el común proceso cognitivo de un negociador le hará desconfiar y creer que la contraparte no se ha comprometido a nada significativo ${ }^{54}$.

53 Thompson (2012), p. 42.

${ }^{54}$ Mnookin \& Ross (1995), p. 15. Se ha señalado que uno de los errores del Reino Unido en las negociaciones de Brexit fue de mantenerlas bajo secreto. La ex Primera Ministra equi- 
En Chile la desconfianza se ha materializado como un problema social. Diversos estudios realizados por el PNUD, han dejado en evidencia que el $60 \%$ de los chilenos manifiesta desconfianza en el otro ${ }^{55}$. De hecho, ya en 2010, la revista Qué Pasa daba a conocer el resultado de una encuesta realizada por Falcon Management y el Centro de Negociación de la Universidad Católica, en que se concluyó que los ejecutivos de grandes empresas del país eran desconfiados y solían ocultar información a sus interlocutores. Solo el $47 \%$ de los ejecutivos creía en la palabra de su contraparte ${ }^{56}$, y estas cifras no parecen mejorar con los años.

Científicos han demostrado que los negociadores que intercambian información reconsideran sus percepciones respecto de los intereses y motivaciones de la otra parte. Luego de un aprovisionamiento recíproco de información, el juicio de los negociadores sobre el otro lado de la mesa tiende a ser más acertado $^{57}$. De este modo, el intercambio de información crea menos espacio para la desconfianza, el engaño y la atribución equívoca sobre los actos de la contraparte ${ }^{58}$.

\section{Comportamientos estratégicos}

Aunque los negociadores dispusieran de toda la información que requieran sobre el objeto de la negociación, el deseo de obtener una porción más grande de la torta puede llevar a las partes a actuar en contra de la obtención de resultados eficientes. De hecho, aquellas conductas, conocidas en la literatura como comportamientos estratégicos u oportunistas, pueden significar un escenario de bloqueo (sin que se alcance ningún acuerdo) o de negociaciones más costosas y prolongadas; reduciendo de aquel modo el tamaño de la torta ${ }^{59}$.

Los comportamientos estratégicos son aquellos empleados para moldear la percepción de la otra parte respecto de los posibles acuerdos que pueden ser alcanzados, es decir, hacer creer que se tiene un mayor o menor RV. Dicho de otro modo, cada parte evalúa cuanto es lo menos o lo máximo que el otro lado estará dispuesto a aceptar, tratando de presionar a la contraparte lo más posible. Normalmente, la primera y más básica técnica utilizada por los negociadores para producir este efecto en la contraparte, es ofertar ampliamente sobre su RV

vocadamente sugirió que "cada reporte de la prensa hará más dificultoso obtener el acuerdo ideal. Todos aquellos que nos insisten en revelar más información, no estarán actuando en el interés nacional”. MarTiLl \& Staiger (2018), p. 17.

${ }^{55}$ Rodríguez (2018), pp. 12-13.

${ }^{56}$ Qué Pasa (2010).

${ }^{57}$ Thompson \& Hastie (1990), pp. 102-103.

${ }^{58}$ MnOokin \& Ross (1995), p. 16. El profesor Marwan Sinaceur ha agregado que la búsqueda de información aumenta las posibilidades de generar ganancias en un proceso de negociación, independientemente de la información que haya sido proporcionada. La creación de ganancias aumenta incluso cuando solo un lado de la mesa es el que busca mayor información. SinACEUR (2010), p. 544.

${ }^{59}$ MnOokin (2011), pp. 284-285. 
y así distorsionar la estimación de la ZOPA (en términos coloquiales, ofertar con el "tejo pasado"). En efecto, los primeros números ofrecidos por una de las partes son procesados de manera inconsciente por la otra, ejerciendo -aun siendo sabido que la primera oferta será desproporcionada - una gran influencia en lo que estimará que la otra parte quiere. Es lo denominado en psicología como el efecto de anclaje (anchoring effect), el que explica que las personas se aproximan a la estimación de un valor con un sesgo numérico ${ }^{60}$. Científicos han demostrado que la estimación final de un valor es influenciada por el número inicialmente proporcionado. Es decir, personas que han recibido en primer término un número relativamente alto como estándar para realizar la determinación del valor final, concluyen estimaciones numéricas más altas que los que reciben un número más bajo como estándar de comparación ${ }^{61}$.

Pero emplear el anclaje trae aparejados diversos riesgos. Así, si la primera oferta es muy agresiva o alta, la contraparte puede que prefiera levantarse de la mesa sin intención de volver. Si la oferta es irrealista, el otro lado lo notará y no la tomará en cuenta. Si luego de una primera oferta, la parte que la realiza la modifica o se retracta a un menor valor, se generará un costo de credibilidad. Por ejemplo, en el contexto de las negociaciones de Brexit, exigencias tajantes como "Brexit significa Brexit", realizadas con el objetivo de moldear la percepción de la Unión Europea, de que el objetivo del Reino Unido era obtener una soberanía política completa, pero con todos los beneficios de un acceso total al mercado comunitario, fueron compromisos poco constructivos y creíbles del Reino Unido ${ }^{62}$. En efecto, el proceso de salida del Reino Unido ya ha sido, a la fecha de escritura de este trabajo, pospuesto tres veces, sin que exista fecha cierta (y seria) para concretar su exclusión de la Unión Europea.

Tácticas empleadas con este objetivo usualmente tienden a ser engañosas y deshonestas ${ }^{63}$, causando malos entendidos entre las partes, reforzando el prejuicio y provocando reacciones que producen contra reacciones en un círculo vicioso ${ }^{64}$. Todo aquello trae como resultado que los negociadores comiencen una competencia enfocada en sus propios intereses, donde el examen racional de posibles soluciones se torna prácticamente imposible ${ }^{65}$. Debe tenerse presente que un quiebre en la confianza existente entre las partes

${ }^{60}$ STrack \& Mussweiler (1997), pp. 437-446.

${ }^{61}$ Esta conclusión fue obtenida en uno de los más conocidos estudios sobre el efecto de anclaje llevado a cabo por TvERSKY y KAHNEMAN (1974), pp. 1124-1131. En el experimento, los científicos entregaron a los participantes de la investigación un número aleatorio entre 0 y 100 , y se les solicitó que indicaran si el porcentaje de naciones africanas en las Naciones Unidas era mayor o menor que ese número. Luego, los participantes debían estimar el porcentaje real.

${ }^{62}$ EidenMüller (2017), p. 56.

${ }^{63}$ Mnookin et al. (2000), p. 23.

${ }^{64}$ Fisher \& URY (2012), p. 19.

${ }^{65}$ Tal como Howard Raiffa ha enseñado, si tú eres muy codicioso o tu adversario es muy codicioso, o si ambos lo son, ambas partes fallarán en alcanzar un acuerdo que pudiera significar ganancias para ambos. RAIFFA (2003), p. 33. 
puede traer consecuencias sumamente perjudiciales a la mesa de negociación. El resultado obvio, la desconfianza, puede que nunca sea superada. La confianza es un commodity muy frágil: difícil de generar, fácil de destruir y a veces imposible de reconstruir. Lo cierto es que se debe tomar en consideración que la pregunta no es solo si es posible confiar en el otro lado, sino que, también, dejar en claro que nuestro lado es igualmente honesto. Cualquier mecanismo utilizado para estos efectos debe ser recíproco, de otra manera, las personas se sentirán obligadas a hacer trampa, porque aquello se esperará que los otros lo hagan. Lo dicho revela entonces, porqué menos de la mitad de los ejecutivos de las empresas chilenas confían en su contraparte.

De este modo, se ha explicado qué comportamientos más cooperativos realzan la habilidad de las partes para descubrir intereses complementarios. Evitar comportamientos estratégicos permite a los negociadores determinar el verdadero valor de los intereses disputados que se están negociando ${ }^{66}$.

\section{Manejando los PROBlemas \\ EN EL PROCESO DE NEGOCIACIÓN}

Como ya fuera indicado, los problemas antes desarrollados no tienen una solución definitiva. Los negociadores deben aprender a manejarlos con la 132 finalidad de no pasar por alto oportunidades que pueden generar ganancias a través de la negociación. Con este propósito, en lo que sigue, son sugeridas tres posibles técnicas que pueden ayudar a las partes a mejorar el diseño de sus negociaciones a través de la creación de confianza y cooperación.

\section{A. Apelar a normas procedimentales que entreguen a las partes un trato justo e igualitario}

No importa cuán conscientes sean los negociadores respecto de los problemas antes desarrollados, es aún posible que las partes no sean capaces de encontrar una solución satisfactoria al momento de distribuir las ganancias creadas o, en definitiva, llegar a acuerdo alguno. Normalmente, esto ocurre debido a que el otro lado de la mesa piensa que no está siendo tratado de forma justa durante las negociaciones ${ }^{67}$.

Se ha argumentado que ofertas verdaderamente justas son aquellas que no toman ventajas de las asimetrías de información mantenida entre las partes. Una oferta, de este modo, será alta (o conforme las expectativas de la otra parte), no porque uno de los negociadores sabe que el otro lado dispone de

\footnotetext{
${ }^{66}$ Mendenhalt (1996), p. 86.

${ }^{67}$ Aquello explica, por ejemplo, por qué la Unión Europea cuidadosamente se aproximó a las negociaciones de Brexit indicando: "Europa será transparente y justa en las conversaciones sobre el Brexit". BARNIER (2017).
} 
la información que le permitirá descubrir una propuesta engañosa con un número menor. En su lugar, una oferta debería ser realizada así (acorde los intereses del otro lado de la mesa) simplemente porque es justa. Empero, científicos han concluido que los oferentes tienden a actuar de manera codiciosa, poniendo en marcha comportamientos estratégicos u oportunistas ${ }^{68}$. Estos resultados, no obstante, son hasta cierto punto lógicamente humanos, porque los negociadores deben buscar resultados ambiciosos que sirvan a sus intereses, sin embargo, aquellos no pueden ser irrealistas al punto de frustrar las negociaciones ${ }^{69}$.

La solución, entonces, debe ser buscada a través de un procedimiento que pueda ser aplicado al momento de repartir la torta. Debe tenerse presente que, la respuesta a qué es lo justo, no debe ser buscada solamente en los números, sino en el "por qué" aquel número es ofrecido al otro lado. De allí que, durante el proceso de negociación, propuestas con una referencia a estándares que se encuentran fuera del control de las partes, tales como valores de mercado, lo dictaminado por la jurisprudencia, las prácticas de la industria, la lex artis profesional, los descubrimientos científicos o el imaginar cómo podría fallar un tribunal, permitirá a los negociadores encontrar una forma objetiva de resolver el problema de distribución.

$\mathrm{Al}$ dar razones objetivas, existe un menor riesgo de que una de las partes sienta que le han sacado provecho y que luego intente repudiar el acuerdo ${ }^{70}$. En estos casos, nadie desistirá o parecerá que está renunciando a sus intereses. Ambos lados serán considerados razonables, por encontrarse aplicando un verdadero balance entre comportamientos cooperativos y competitivos ${ }^{71}$.

\section{B. Mejorando los acuerdos adoptados: alcanzar arreglos posteriores al acuerdo}

Ha sido establecido de forma científica que, si el acuerdo alcanzado no es un óptimo de Pareto, siempre existe espacio para mejorarlo ${ }^{72}$. Howard Raiffa postuló ya hace varios años el papel de un "embellecedor de contratos", quien es un tercero que realiza un análisis de transacción ya cerrada, con el objetivo de ofrecer a las partes posibles mejoras al mismo ${ }^{73}$. En la actualidad, esta estrategia se ha denominado como un acuerdo tras el acuerdo (postsettlement settlement), y puede ser realizada por las mismas partes o, como fuera originalmente planteada, involucrando a un tercero.

\footnotetext{
${ }^{68}$ Pillutla \& Murnighan (1995), pp. 1410-1417.

${ }^{69}$ MNookin et al. (2000), p. 34.

${ }^{70}$ Fisher \& URY (2012), p. 84.

${ }^{71}$ Op. cit., p. 85.

${ }^{72}$ Teich et al. (1996), p. 537.

${ }^{73}$ Raiffa (2003), p. 221. Esto ha sido ilustrado por Howard Raiffa con el caso denominado la "justa división de una colección de arte" (Fair Division of an Art Collection). RaIFFA et al. (2007),
} pp. 338-340. 
En este escenario, el acuerdo ya alcanzado se transforma en el nuevo BATNA de las partes, el que puede ser utilizado para explorar su reemplazo. En efecto, las partes pueden seguir negociando e intercambiando libremente más información que la entregada al momento de negociar el acuerdo original, pues, mal que mal, ya han llegado a un arreglo. Si no es posible llegar a un nuevo acuerdo, las partes simplemente pueden mantener el inicial. Siguiendo a Leight Thomson, esta estrategia permite a los negociadores mover a un acuerdo en que todos ganan desde un nivel 1 a un nivel 2 o 3 , conforme con la clasificación señalada más atrás ${ }^{74}$.

Para ejemplificar, se asumirá que las partes (A y B) se encuentran negociando sobre la liquidación (disolución) de una sociedad. A y B tienen una distinta avaluación sobre las maquinarias de la empresa a distribuir. En el primer escenario, cada parte está dispuesta a pagar la mitad de lo exigido por la otra:

\begin{tabular}{|c|c|c|c|c|}
\hline Maquinarias & Valoración para A & Valoración para B & Cuota de A & Cuota de B \\
\hline 1 & $\$ 1000000$ & $\$ 4000000$ & $\$ 500000$ & $\$ 2000000$ \\
\hline 2 & $\$ 8000000$ & $\$ 2000000$ & $\$ 4000000$ & $\$ 1000000$ \\
\hline 3 & $\$ 6000000$ & $\$ 4000000$ & $\$ 3000000$ & $\$ 2000000$ \\
\hline & & Totales & $\$ 7500000$ & $\$ 5000000$ \\
\hline & & & $\begin{array}{c}\text { Valor creado } \\
\text { por el acuerdo }\end{array}$ & $\$ 12500000$ \\
\hline
\end{tabular}

¿De qué manera las partes pueden distribuir y asignar las maquinarias para generar mayores ganancias?

\begin{tabular}{|c|c|c|c|c|}
\hline Maquinarias & Valoración para A & Valoración para B & Cuota de A & Cuota de B \\
\hline 1 & $\$ 1000000$ & $\$ 4000000$ & & $\$ 4000000$ \\
\hline 2 & $\$ 8000000$ & $\$ 2000000$ & $\$ 8000000$ & \\
\hline 3 & $\$ 6000000$ & $\$ 4000000$ & & $\$ 4000000$ \\
\hline & & Totales & $\$ 8000000$ & $\$ 8000000$ \\
\hline & & & $\begin{array}{c}\text { Valor creado } \\
\text { por el acuerdo }\end{array}$ & $\$ 16000000$ \\
\hline
\end{tabular}

Supóngase que el acuerdo anterior fue cerrado y se transformó en el nuevo BATNA de los negociadores. Las partes se encuentran conformes con sus ganancias y la asignación de las maquinarias. No obstante, ¿ es posible buscar una distribución más eficiente?

${ }^{74}$ Thompson (2012), p. 107. Véase supra, apartado II. A. 


\begin{tabular}{|c|c|c|c|c|}
\hline Maquinarias & Valoración para A & Valoración para B & Cuota de A & Cuota de B \\
\hline 1 & $\$ 1000000$ & $\$ 4000000$ & & $\$ 4000000$ \\
\hline 2 & $\$ 8000000$ & $\$ 2000000$ & $\$ 8000000$ & \\
\hline 3 & $\$ 6000000$ & $\$ 4000000$ & $\$ 6000000$ & \\
\hline & & Totales & $\$ 14000000$ & $\$ 4000000$ \\
\hline & & $\begin{array}{c}\text { Valor creado } \\
\text { por el acuerdo }\end{array}$ & $\$ 18000000$ \\
\hline
\end{tabular}

En efecto, la nueva propuesta genera mayor valor y, por lo demás, resulta equitativo si son analizados los pagos complementarios para distribuir las ganancias:

\begin{tabular}{|l|c|c|}
\hline & A & B \\
\hline Maquinarias & $\$ 14000000$ & $\$ 4000000$ \\
\hline Pagos & $-\$ 5000000$ & $\$ 5000000$ \\
\hline Subtotal & $\$ 9000000$ & $\$ 9000000$ \\
\hline Valor del acuerdo & & $\$ 18000000$ \\
\hline
\end{tabular}

Tomemos ahora un ejemplo sacado de la realidad, relativo a las negociaciones de Brexit realizadas respecto de la frontera aduanera y migratoria a ser establecida entre la República de Irlanda e Irlanda del Norte (Irish backstop). Aquellas fueron infructuosas a pesar de que el Reino Unido y la Unión Europea estaban de acuerdo de que su implementación era indeseada. Actualmente, con o sin acuerdo, la frontera tendrá lugar. Sin embargo, concluida la salida del Reino Unido de la Unión Europea, ya sea cerrado o no el acuerdo de Brexit sin aquella consideración, las partes, conforme a sus aparentes intereses, no deberían tener dificultes en reabrir el asunto y obtener una mejor solución en este punto.

\section{Construir cooperación: tit for tat}

Siguiendo la investigación de Robert Axelrod ${ }^{75}$, se ha señalado en la literatura que comportamientos estratégicos como mimetismos y de espejo, puede hacer que las partes descubran resultados integradores ${ }^{76}$. Bajo la lógica de este autor, los negociadores deberían realizar una apertura condicional en el sentido de comenzar con actos cooperativos a través de pequeños intentos de creación de ganancias conjuntas. Luego, la estrategia consiste en adoptar exactamente el mismo paso realizado por la contraparte: si la actuación es recíproca a un intento de crear ganancia, las partes debería continuar con aquel enfoque. $\mathrm{Si}$

\footnotetext{
${ }^{75}$ Axelrod (1984), pp. 27-54.

${ }^{76}$ Thompson (2012), p. 137.
} 
la contraparte no se comporta de igual manera, las partes deberían detener los intentos cooperativos. Sin embargo, si la otra parte intenta reestablecer la cooperación, el otro negociador debería reciprocar: respuesta equivalente o "toma y daca" (Tit for Tat).

De este modo, a través del Tit for Tat, los negociadores pueden construir cooperación y confianza en aquellos intentos en que las partes actúan recíprocamente, mientras al mismo tiempo, se protegen de movimientos competitivos y engañosos y del aprovechamiento del otro lado de la mesa al detener la colaboración.

\section{Conclusiones}

El conflicto es inevitable, pero una resolución justa y eficiente del mismo no lo es ${ }^{77}$. Sin embargo, para evitar los acuerdos en que todas las partes salen perdiendo (lose-lose agreements), los negociadores deben mejorar su percepción y juicio sobre los intereses que se tienen del otro lado de la mesa. Un serio error ocurre cuando las partes entran a la negociación con una mentalidad de suma cero respecto de los conflictos en juego; asumiendo que las preferencias de la contraparte se encuentran siempre en contraposición ${ }^{78}$. Aquello explica por qué los negociadores tienden a no reconocer cuando estos tienen 136 intereses que resultan compatibles; cerrando acuerdos que, en definitiva, las partes estiman indeseables en contraste con otras soluciones disponibles sobre la mesa.

Las diferencias, en efecto, pueden traer ganancias para las partes. Aquellas constituyen precisamente el rango dentro del cual los negociadores pueden encontrar intercambios que los puedan dejar en una mejor posición. Pero para lograr esto, el intercambio de información no es solo necesario, sino que, también, se ha probado científicamente como un medio que minimiza el espacio para la desconfianza, el engaño y la equívoca atribución negativa a los actos de la contraparte. Entender los intereses del otro lado de la mesa trae consigo comportamientos cooperativos, que pueden aumentar la habilidad de los negociadores para descubrir intereses complementarios.

Resolver el problema de la otra parte no es solo su problema. Un acuerdo en que todas las partes ganan (win-win agreements) será posible y sostenible en el tiempo solo si también alcanza al menos el BATNA del otro lado. Abandonar comportamientos estratégicos por ofertas justas y procesos de distribución objetivos, posibilitará a los negociantes cumplir sus metas de manera efectiva $\mathrm{y}$, al mismo tiempo, permitirá protegerse de jugadas oportunistas.

\footnotetext{
${ }^{77}$ Mnookin (1993), p. 235.

${ }^{78}$ Thompson \& Reid (1990), p. 101.
} 


\section{BiBLIOGRAFÍA}

AxeLrod, Robert (1984). The evolution of cooperation. New York: Basic Books Publishers.

Bazerman, Max H. \& Don A Moore (2013). Judgment in Managerial Decision Making. $8^{\text {th }}$ ed., New Jersey: Wiley.

Barnier, Michel (2017). Europe Will Be Transparent and Fair in Brexit Talks. In Financial Times. Disponible en www.ft.com/content/fd5ebab0-10b7-11e7a88c-50ba212dce4d [fecha de consulta: 12 de marzo de 2019].

EIdenmÜlLER, Horst (2016). "Negotiating and Mediating Brexit". Pepperdine Law Revier. N. ${ }^{\circ}$ 39. Malibu.

EIDENMÜLLER, Horst (2017). Brexit Negotiations Series: "How to Negotiate a Successful Brexit. Oxford Business Law Blog. Disponible en www.law.ox.ac.uk/ business-law-blog/blog/2017/05/brexit-negotiations-series-how-negotiatesuccessful-brexit [fecha de consulta: 6 de marzo de 2019].

Fisher, Roger \& William URY (2012). Getting to Yes. Negotiating an agreement without giving in. $3^{\text {rd }}$ ed. Cambridge, Massachusetts: Random House Business Books.

Korobkin, Russel \& Chris Guthrie (2006). "Heuristics and Biases at the bargaining table", in Carrie J. Menkel-Meadow, Andrea Kupfer Schneider and Lela Porter Love (eds.). Negotiation. Processes for Problem Solving. New York: Aspen Publishers.

Lax, David \& James Sebenius (1986). The Manager as Negotiator: Bargaining for Cooperation and Competitive Gain. New York: Free Press.

Lax, David \& James Sebenius (2006). 3-D Negotiation. Powerful Tools to Change the Game in Your Most Important Deals. Boston: Harvard Business School Press.

Mendenhalt, Robert (1996). "Post-Settlement Settlements: Agreeing to Make Resolutions Efficient”. Journal of Dispute Resolution. N. ${ }^{\circ}$ 81. Jefferson City.

Martill, Benjamin \& Uta Staiger (2018). "Cultures of Negotiation: Explaining Britain's hard bargaining in the Brexit negotiations". Disponible en www. dahrendorf-forum.eu/wp-content/uploads/2018/09/Cultures-of-Negotiation-3.pdf [fecha de consulta: 15 de marzo de 2019].

Mnookin, Robert (1993). "Why Negotiations Fail: An Exploration of Barriers to the Resolution of Conflict". The Ohio State Journal on Dispute Resolution. Vol. 8, n. ${ }^{\circ}$ 2. Ohio.

Mnookin, Robert \& Lee Ross (1995). "Introduction”, in Kenneth ArRow, Robert Mnookin, Lee Ross, Amos Tversky and Robert Wilson (eds.). Barriers to Conflict Resolution. Stanford: W. W. Norton \& Company.

Mnookin, Rober, Scott Peppet \& Andrew Tulumello (2000). Beyond Winning. Negotiating to create value in deals and disputes. $2^{\text {nd ed }}$. London: The Belknap Press of Harvard University Press.

Mnookin, Robert (2011). "Bargaining with the Devil: When to Negotiate, When to Fight", in Jay FolBerg and Dwight Golann (eds.). Lawyer Negotiation. Theory, Practice, and Law. New York: Wolters Kluwer. 
Nadler, Janice, Leight Thompson \& Leaf van Boven (2003). "Learning Negotiation Skills". Management Science. Vol. 49, n. ${ }^{\circ}$. Catonsville.

Patton, Bruce (2005). "Negotiation", in Michael L. MoffitT and Robert C. BorDOne (eds.). The Handbook of Dispute Resolution. San Francisco: Jossey-Bass, Harvard Law School.

Pillutla, Madan \& Keith Murnighan (1995). "Being fair or appearing fair: Strategic behaviour in ultimatum bargaining". Academy of Management Journal, Vol. 38, n. ${ }^{\circ}$ 5. Briarcliff Manor.

RAIFFA, Howard (2003). The art and science of negotiation. How to resolve conflicts and get the best out of bargaining. London: Harvard University Press.

Raiffa, Howard, John Richardson \& David Metcafe (2007). Negotiation Analysis. The Science and Art of Collaborative Decision Making. London: The Belknap Press of Harvard University Press.

Reuters (2017). "Text of PM May's letter to EU's Tusk triggering Brexit process". Disponible en: https://uk.reuters.com/article/uk-britain-eu-letter-textidUKKBN1701JH [fecha de consulta: 6 de marzo de 2019].

Revista QuÉ PASA (2010). "Así negocian los chilenos". Disponible en www.quepasa.cl/articulo/negocios/2010/06/16-3587-9-asi-negocian-los-chilenos.shtml/ [fecha de consulta: 23 de abril de 2019].

Rodríguez, Darío (2018). "Confianza en Chile, un preciado (y escaso) bien social”. Anuario de Negociación y Resolución de Conflictos. N. ${ }^{\circ}$ 2, Santiago.

Schaerer, Michael, David Loschelder \& Roderick SwaAb (2016). "Bargaining zone distortion in negotiation: the elusive power of multiple alternatives". Organizational Behaviour and Human Decision Processes. N. ${ }^{\circ} 137$. Boston.

Schwarz, Sabine, Markus Voeth \& Uta Herbst (2010). "Information asymmetry in buyer-seller negotiations and its impact on effectiveness, efficiency and satisfaction". 26th IMP-conference in Budapest. Budapest.

SinAceur, Marwan (2010). "Suspending judgment to create value: Suspicion and trust in negotiation”. Journal of Experimental Social Psychology. n. ${ }^{\circ} 46$. Canterbury.

STRACK Fritz \& Thomas Mussweiler (1997). "Explaining the enigmatic anchoring effect: Mechanisms of selective accessibility". Journal of Personality and Social Psychology. N. ${ }^{\circ}$ 73. Washington.

Teich, Jeffrey E., Hannele Wallenius, Jyrki Wallenius and Stanley Zionts (1996). "Identifying Pareto-Optimal settlement for two-party resource allocation negotiations". European Journal of Operation Research. N. ${ }^{\circ} 93$. Amsterdam.

Thompson, Leight (2012). The Mind and Heart of the Negotiator. $5^{\text {th }}$ ed. International Edition. London: Pearson.

Thompson, Leigh \& Reid Hastie (1990). “Social perception in negotiation”. Organizational Behaviour and Human Decision Process. N. ${ }^{\circ}$ 47, Issue 1. Amsterdam.

Thompson, Leight \& Dennis Hrebec (1996). "Lose-Lose Agreements in Interdependent Decision Making". Psychological Bulletin. Vol. 120, n. ${ }^{\circ} 3$. Washington. 
Tversky, Amos \& Daniel Kahneman (1974). "Judgment under uncertainty: Heuristics and biases". Science. N. ${ }^{\circ} 185$. Washington.

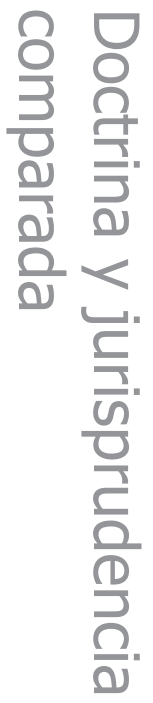

